\title{
Parametric Gain in the Strongly Nonlinear Regime and Its Impact on 10-Gb/s NRZ Systems With Forward-Error Correction
}

\author{
Paolo Serena, Alberto Bononi, Jean-Christophe Antona, and Sébastien Bigo, Member, IEEE
}

\begin{abstract}
In this paper, we show that the nonlinear parametric gain (PG) interaction between signal and noise is a nonnegligible factor in the design and analysis of long-haul dispersion-managed optical 10-Gb/s ON-OFF keying nonreturn to zero transmission systems operated at small signal-to-noise ratios (OSNRs) such as those employing forward-error correction (FEC) coding. In such a regime, we show that the in-phase noise spectrum exhibits a large gain close to the carrier frequency, which is due to the higher order noise terms accounting for the noise-noise beating during propagation that is usually neglected in the nonlinear Schrödinger equation. With a novel stochastic analysis that keeps such higher order terms, we are able to analytically quantify the maximum tolerable signal power after which PG unacceptably degrades system performance. We verify such an analytical power threshold by both simulation and experiment. We finally quantify the needed extra OSNR, or equivalently FEC coding gain, required when taking PG into account.
\end{abstract}

Index Terms-Karhunen-Loéve expansion, Kerr effect, modulation instability, parametric gain.

\section{INTRODUCTION}

A NY optical signal of sufficiently large power propagating in a single-mode fiber interacts with optical noise through a four-wave mixing (FWM) process; on that account, the noise experiences a frequency-dependent gain. This phenomenon is known as parametric gain (PG), and in the anomalous propagation regime it may cause modulation instability [1]. The PG process can be positively exploited to build parametric amplifiers and wavelength converters in short highly nonlinear fibers [2], [3]. However, for optical transmission systems, it is a potential source of system degradation because of the signal interaction with the amplified spontaneous emission (ASE) noise. The problem has been traditionally tackled through a smallsignal approach to the nonlinear Schrödinger equation (NLSE) [1], [4], [5]. Whenever the received optical signal-to-noise ratio (OSNR) is large enough, the signal field is little affected by noise during propagation so that the propagated signal can be evaluated without ASE. The small-signal model consists of a linearization of the NLSE around the known noiseless signal

Manuscript received March 15, 2004; revised December 2, 2004.

P. Serena and A. Bononi are with the Dipartimento di Ingegneria dell'Informazione, Universitá degli Studi di Parma, 43100 Parma, Italy (e-mail: serena@tlc.unipr.it).

J.-C. Antona and S. Bigo are with Alcatel Research and Innovation, 91460 Marcoussis, France (e-mail: Jean-Christophe.Antona@ alcatel.fr).

Digital Object Identifier 10.1109/JLT.2005.850809 field, which leads to two linear differential equations relating the real (in-phase) and imaginary (quadrature) components of the ASE noise [5]. Such a system can be solved exactly in terms of Bessel functions when the signal is a continuous wave (CW) [4], [5]. In the small-signal model, the initially white Gaussian ASE thus remains Gaussian along the propagation and changes its power spectral density (PSD), which becomes frequency dependent, i.e., colored. Starting from the smallsignal model, Bosco et al. [6] evaluated the impact of PG on the bit error rate (BER) of an ON-OFF keying (OOK)modulated signal by an exact analysis of the optical quadratic detection process [7] (thereby avoiding the usual Gaussian approximation for BER evaluation) and by calculating the decision variable statistics by expanding the PG-colored ASE on a signal-dependent Karhunen-Loéve (KL) basis. Unfortunately, such a model ignores the intersymbol interference (ISI) that inevitably affects a modulated (non-CW) signal. An improved model, which accounts for ISI while still adopting the smallsignal PG model, was introduced by Holzlöhner et al. [8], where they were able to evaluate the BER through a covariance matrix method. However, as pointed out in [8], the smallsignal model may fail when the noise is far from small with respect to the signal, as for instance in the tails of the noise probability density function (pdf) of the decision variable, especially when the system is operated at small OSNR. Another situation in which the small-signal model is clearly inappropriate occurs in systems operating close to the zero-dispersion wavelength [9].

In [10], the limits of the small-signal model were investigated by using a semianalytical BER evaluation method similar to the one in [6] that utilizes the noise PSD estimated by Monte Carlo simulations of propagation of a CW plus ASE. At small powers, the results of the Monte Carlo-estimated PSD coincide with the predictions of the small-signal model. After a specific power threshold, instead, the simulated PSD of the in-phase ASE component (the most important for the BER evaluation of OOK systems [6]) tends to rapidly and substantially inflate in the low frequency band, a feature not captured by the small-signal model. Such a feature, which dramatically affects system performance, is due to the quadratic and higher order nonlinear noise-noise beating terms that must therefore be included in the NLSE for a correct ASE PSD estimation.

Our first objective in this paper is to develop a stochastic analysis of the NLSE beyond the small-signal model. Since 
the results in [10] have shown that the ASE PSD grows up very quickly after a specific power, we are interested in an analytical expression of the power threshold at which the effect appears. With this in mind, we focus our attention on the zero frequency (i.e., the central wavelength of the signal spectrum in its low-pass representation) of the in-phase ASE PSD. At such a particular frequency, a perturbative stochastic analysis of the NLSE is feasible and leads to an expression of the in-phase ASE PSD inflation, from which we get the main analytical result of the paper, a formula of the PG power threshold.

We then test our formula against both simulations and experimental results on a $15 \times 100 \mathrm{~km} \mathrm{10-Gb/s} \mathrm{OOK} \mathrm{nonreturn-}$ to-zero (NRZ) Teralight-based system in which an extra noise source is inserted at each amplifier along the line in order to control the amount of PG. For such a system, we observe a strong correlation between the OSNR penalty and the ASE in-phase PSD inflation, which both sharply increase when the transmitted power is close to the power threshold predicted by our formula.

The second objective of this paper is then to assess whether practical long-haul systems, working with forward-error correction (FEC) coding at small OSNR, fall in the strongly nonlinear PG regime or not, and to quantify the incurred OSNR/FEC coding gain penalty with respect to what one would predict by neglecting PG. To this aim, extensive use will be made of the semianalytical BER evaluation model developed in [10].

The paper is organized as follows. In Section II, we introduce the system model and describe the main steps of the semianalytical BER evaluation. In Section III, the small-signal model is studied and its limits in the strongly nonlinear regime are checked both by simulation and by experiment. Section IV contains a novel stochastic analysis of the NLSE that leads to the explicit power threshold formula. The mathematical details are given in the appendixes. Section $\mathrm{V}$ discusses the system implications of PG in long-haul systems. Finally, the main conclusions are drawn in Section VI.

\section{The System Model}

The propagation of the normalized electric field $A(z, t)$ in a single-mode optical fiber link composed of $N$ identical fiber spans, $z$ being the distance and $t$ the time in the retarded signal frame, is described by the NLSE

$$
\frac{\partial A}{\partial z}=j \frac{\beta_{2}(z)}{2} \frac{\partial^{2} A}{\partial t^{2}}-j \gamma(z)|A|^{2} A e^{g(z)}+S(z, t)
$$

where $\beta_{2}(z)$ is the chromatic coefficient parameter; $\gamma(z)$ is the nonlinear parameter; $g(z) \triangleq \sum_{k} g_{k} \mathrm{U}\left(z-z_{k}\right)-\int_{0}^{z} \alpha(\zeta) \mathrm{d} \zeta$ is the total logarithmic gain up to $z$, with $\alpha(z)$ being the fiber attenuation and $G=e^{g_{k}}$ the power gain of the $k$ th lumped amplifier placed at the end of the $k$ th fiber span, and the unit step function is defined as $\mathrm{U}(z)=1$ for $z>0$ and 0 otherwise; $S(z, t) \triangleq \sum_{k} n\left(z_{k}, t\right) \delta\left(z-z_{k}\right)$ is the ASE noise generated by the lumped amplifiers, where $n\left(z_{k}, t\right)$ are indepen- dent identically distributed (i.i.d.) complex Gaussian random processes with $\mathrm{E}\left[n\left(z_{k}, t\right)\right]=0$ and $\mathrm{E}\left[n\left(z_{k}, t\right) n^{*}\left(z_{l}, t+\tau\right)\right]=$ $N_{\mathrm{a}} \delta(\tau) \delta_{\mathrm{kl}}$, with $\delta(\tau)$ being the Dirac delta function, $\delta_{\mathrm{kl}}$ the Kronecker delta function, and $N_{\mathrm{a}}$ is the one-sided ASE PSD generated by each amplifier. Equation (1) refers to a single polarization and $S$ is the component of the ASE copolarized with the signal. Polarization effects are neglected in this analysis. For a system with $N$ spans, $N_{\text {a }}$ can be obtained from the linear OSNR (i.e., the one in absence of PG) measurable at the receiver as

$$
N_{\mathrm{a}} \triangleq \frac{\langle P\rangle}{2 N \operatorname{OSNR} \Delta \nu}
$$

where $\Delta \nu \cong 12.5 \mathrm{GHz}$ is the conventional $0.1-\mathrm{nm}$ OSNR measurement noise bandwidth, $\langle P\rangle$ is the time-averaged signal power, which we will express in the following as a fraction $\kappa$ of the peak power $P$, i.e., $\langle P\rangle \triangleq \kappa P$, and the factor 2 is due to the inclusion of the ASE in the orthogonal polarization, which we assume does not interact with the signal and remains white at the receiver.

If the input is a CW signal $A(0, t)=\sqrt{P}$, then the noiseless solution of (1) is $A(z)=\sqrt{P} \exp \left[-j \Phi_{\mathrm{NL}}(z)\right]$, with $\Phi_{\mathrm{NL}}(z) \triangleq P \int_{0}^{z} \gamma(\zeta) e^{g(\zeta)} \mathrm{d} \zeta$ being the cumulated nonlinear phase up to coordinate $z$ [1]. Such a situation corresponds, for instance, to the transmission of a flat-top mark in an OOK NRZ transmission with small pulse distortion during propagation. In the presence of noise, we write the solution of (1) in the form

$$
A(z, t) \triangleq(\sqrt{P}+a(z, t)) e^{-j \Phi_{\mathrm{NL}}(z)}
$$

where $a(z, t) \triangleq a_{r}(z, t)+j a_{i}(z, t)$ is the complex envelope of the cumulated ASE noise, with $a_{r}$ and $a_{i}$ being its in-phase and quadrature components, respectively. When in each span the gain perfectly recovers the loss, the photodetected current corresponding to (3) can be expressed as

$$
I(t)=P+2 \sqrt{P} a_{r}+\left(a_{r}^{2}+a_{i}^{2}\right)
$$

where for simplicity we assumed unit responsivity and ignored the ASE in the orthogonal polarization. The second term on the right-hand side, known as the spontaneous-signal beat noise, is the one that mostly determines the performance of an OOK system, while the last term, known as the spontaneous-spontaneous beat noise, can only increase the received power. If the PSD of the received ASE is known, one can evaluate the corresponding BER by using a KL method for quadratic detectors [6], [8]. The KL method becomes necessary because it was shown that the standard Gaussian BER evaluation method fails in the presence of PG [6]. In this paper, 
we will evaluate the BER using a modified KL method along the lines of [11], in which

1) we use the received OOK signal, solution of (1) in the absence of noise, to evaluate the sampled current at the sampling time;

2) we separately evaluate the PSD of the ASE $a(z, t)$ on marks by solving (1) with (3) by Monte Carlo simulation using the peak transmitted power as the value of $P$ in (3). The ASE PSD on spaces is obtained as in a linear propagation.

The lengthy details of the modified KL method, which includes accurate optical and electrical filtering, are reported elsewhere [12].

These steps, which implement a superposition of the effects of self-phase modulation (SPM) and PG, imply that we ignore the effects of pulse modulation on the ASE noise along the transmission line and are thus expected to be accurate for systems with moderate noiseless signal distortion, where PG is expected to be the dominant impairment. In [8], it is shown how to accurately account for the SPM-PG coupling due to modulation, although in a linearized version of the NLSE. A key novelty in this paper, however, is that we will keep the ASE-ASE beating terms during propagation in the NLSE and show under which circumstances they are responsible of system breakdown.

\section{The SMALl-Signal Model}

The standard approach to the study of PG is a linearization of the NLSE that one obtains by substituting (3) in (1) and dropping all quadratic and higher order terms in $a(z, t)$. In the frequency domain, this leads to

$$
\left\{\begin{array}{l}
\frac{\partial a_{r}(z, \omega)}{\partial z}=\frac{\beta_{2} \omega^{2}}{2} a_{i}(z, \omega)+S_{r}(z, \omega) \\
\frac{\partial a_{i}(z, \omega)}{\partial z}=-\frac{\beta_{2} \omega^{2}}{2} a_{r}(z, \omega)-2 \gamma P a_{r}(z, \omega) e^{g(z)}+S_{i}(z, \omega)
\end{array}\right.
$$

where $a_{r, i}(z, \omega)$ is the Fourier transform of $a_{r, i}(z, t)$, and $S_{r}(z, \omega)$ and $S_{i}(z, \omega)$ are the Fourier transforms of the ASE in-phase and quadrature components, respectively. ${ }^{1}$ The solution of (5) can be expressed in terms of a transfer matrix [5] from which one can evaluate the PSD of the received noise and finally the BER.

Even if the small-signal model can be successfully applied to many practical systems, it is possible to find cases of interest for which it fails. For instance, Marcuse [9] pointed out that systems working at $\beta_{2}=0$, no matter how large the linear OSNR, exhibit a catastrophic build up of the ASE PSD that rapidly destroys the BER. For such systems, the small-signal model (5) erroneously predicts an evolution of $a_{r}$ as in a linear system $(\gamma=0)$, and a nonlinear growth of $a_{i}$ that would imply an eye opening on marks, according to (4), and thus a BER

\footnotetext{
${ }^{1}$ Here and in the rest of the paper, we will use the same letter to denote both a time-domain function and its frequency-domain Fourier transform. The argument of the function, when omitted, will be clear from the context.
}
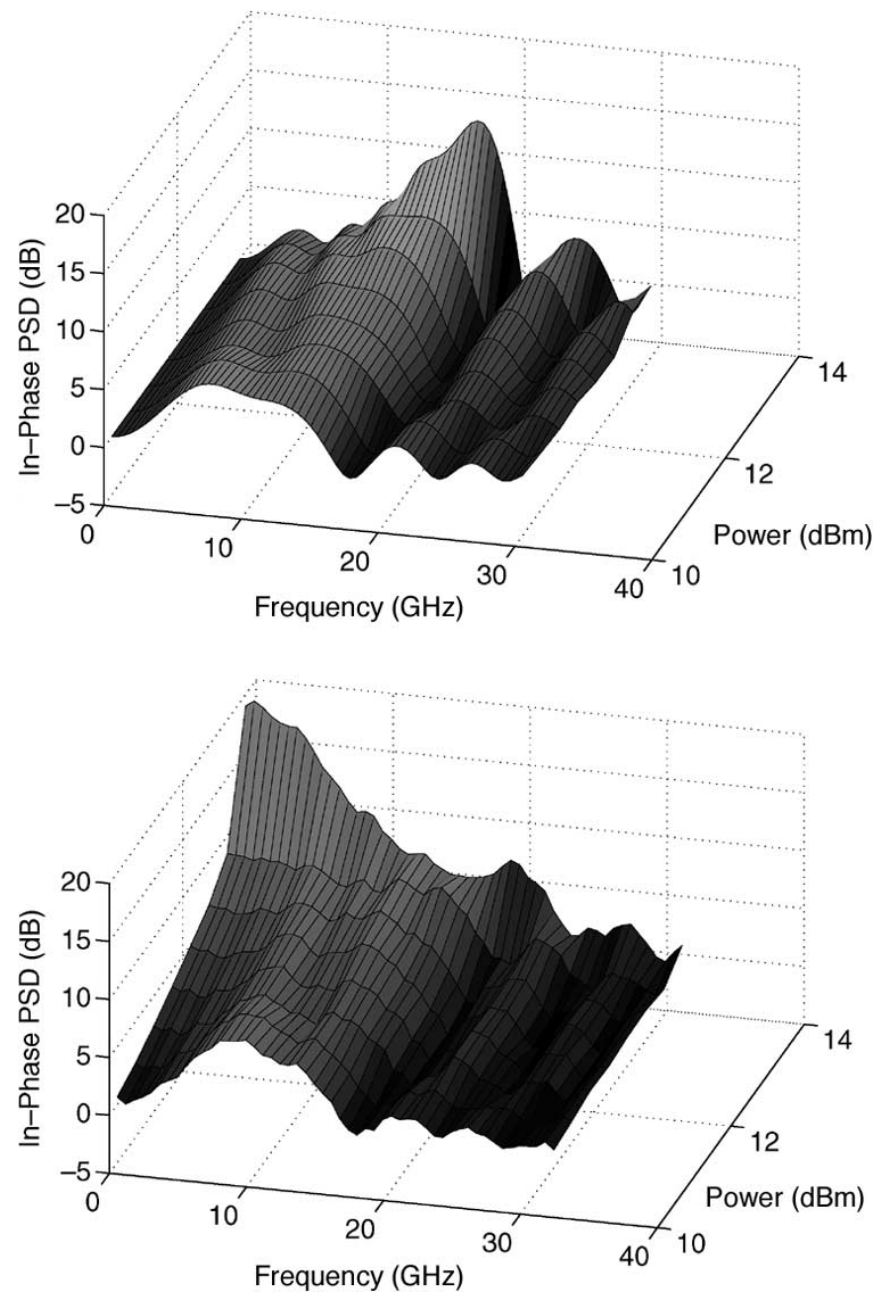

Fig. 1. Normalized in-phase ASE PSD versus frequency and signal power $P$. Top: Small-signal model. Bottom: Monte Carlo simulation. Teralight five-span map with OSNR $=16 \mathrm{~dB}$.

improvement. We will see that practical systems with finite dispersion $\beta_{2}$ behave like systems at zero dispersion when the linear OSNR is sufficiently small.

Since the ASE propagation in (5) depends on the product $\beta_{2} \omega^{2}$, a similar inconsistency of the small-signal model can be observed in its prediction of the ASE PSD at $\omega=0$. In Fig. 1, we show a plot of the PSD of the in-phase ASE component normalized to its value in the absence of $\mathrm{PG}$ versus both frequency and $\mathrm{CW}$ power $P$ for a dispersion-mapped $5 \times 100 \mathrm{~km}$ system with Teralight transmission fiber (dispersion $D=8 \mathrm{ps} / \mathrm{nm} / \mathrm{km}$ and $\gamma=1.7 \mathrm{~W}^{-1} \mathrm{~km}^{-1}$ ), with full inline compensation, and working at an OSNR $=16 \mathrm{~dB}$. The top plot refers to the PSD predicted by the small-signal model while the bottom plot was obtained by estimating the PSD by a direct Monte Carlo simulation of the noise with the averaged periodogram method [13] for a total of $2^{16}$ noise samples. Comparison of the two figures reveals the failure of the smallsignal model in predicting the PSD inflation at low frequencies when the power $P$ is large. We note from (4) that such an inflation has a major impact on BER.

We experimentally verified the effect of the low-frequency PG inflation of the in-phase PSD by analyzing a $15 \times 100 \mathrm{~km}$ Teralight system in a recirculating loop whose setup is 


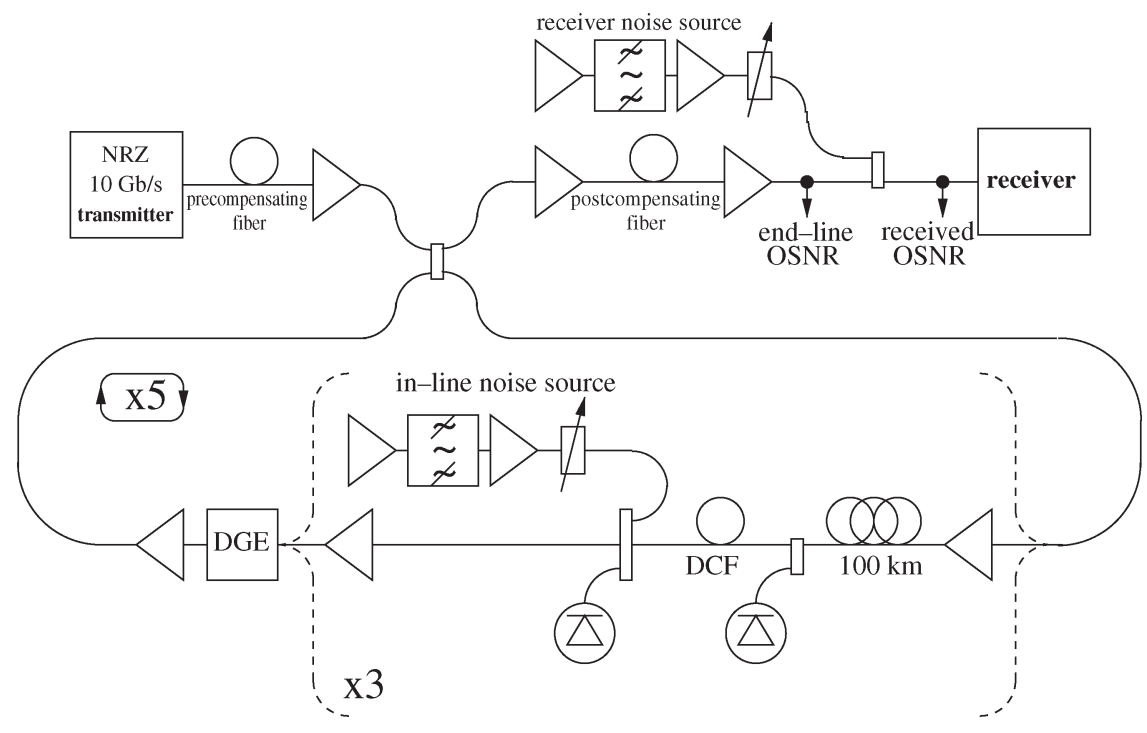

Fig. 2. Experimental setup of a $15 \times 100 \mathrm{~km}$ Teralight system.

described in Fig. 2. We transmitted a signal channel at $\lambda=$ $1545.6 \mathrm{~nm}$, externally OOK modulated at $R=10 \mathrm{~Gb} / \mathrm{s}$ with NRZ supporting pulses, and a $2^{15}-1$ pseudorandom bit sequence (PRBS) with an extinction ratio of $13 \mathrm{~dB}$. Each span is compensated at its end by a dispersion-compensating fiber (DCF) within a dual-stage amplifier with $+30 \mathrm{ps} / \mathrm{nm}$ of residual dispersion after compensation (it will be referred to as inline residual dispersion). The first stage provides Raman amplification within the Teralight while the second stage uses hybrid backward Raman in the DCF and final erbium amplification so as to completely recover the span loss.

In order to let the erbium amplifiers work linearly for the desired signal, we added 13 dummy channels, placed starting at $5 \mathrm{~nm}$ to the right of the signal wavelength, and similarly 13 dummy channels to the left, away enough from the signal so as to avoid any cross nonlinear effects. Optimized pre- and postcompensating fibers were also provided at the transmitter $(-200 \mathrm{ps} / \mathrm{nm})$ and receiver $(+250 \mathrm{ps} / \mathrm{nm})$, respectively [14].

At the end of the loop, a dynamic gain equalizer restored a flat optical spectrum. In front of the receiver, an optical filter of bandwidth $0.23 \mathrm{~nm}$ selected the signal channel while a variable attenuator was used to set the desired BER.

We isolated the effects of SPM from those of PG by toggling ON/OFF an extra inline noise source, also shown in the figure. When ON, we set the end-line OSNR (see figure) to either 16 or $19 \mathrm{~dB}$, while with the noise source off the end-line OSNR was greater than $30 \mathrm{~dB}$, hence with a negligible PG. Since the end-line OSNR was fixed, we measured the received OSNR that yields a $\mathrm{BER}=10^{-5}$ by acting on the final attenuator so as to vary the preamplifier noise figure.

Fig. 3 reports in solid lines the measured received OSNR at $\mathrm{BER}=10^{-5}$ versus the average signal power both without PG (SPM and white ASE) and with PG. When operating at an end-line OSNR $=16 \mathrm{~dB}$, we observe an extra penalty due to a PG of nearly $1 \mathrm{~dB}$ at large power. For comparison, we also report in dashed lines the corresponding results obtained in a numerical simulation where the small-signal model for the ASE PSDs was used. The results of the numerical model are slightly

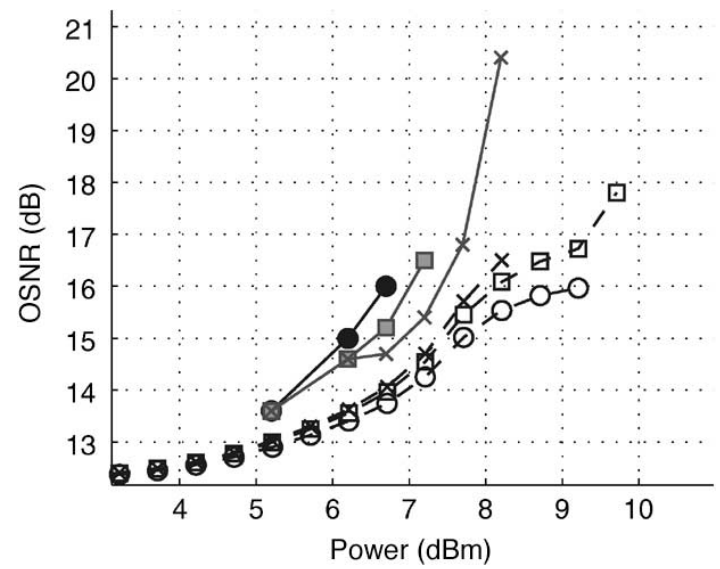

Fig. 3. OSNR versus the average power at a $\mathrm{BER}=10^{-5}$ for the 15 -span Teralight experimental setup. Solid lines: Experimental data. Dashed lines: Numerical results obtained with the small-signal ASE PSD. Circles: Endline OSNR $=16 \mathrm{~dB}$. Squares: End-line OSNR $=19$ dB. Crosses: End-line OSNR $>30 \mathrm{~dB}$ (no PG).

offset for ease of visualization. The important observation is that the small-signal model incorrectly predicts an improvement of performance in the presence of PG.

In order to theoretically investigate the limits of the smallsignal model, in the next section we present an analysis of the NLSE by including the noise-noise beating terms.

\section{The Strongly Nonlinear Regime}

We already observed that the noise-noise beating terms can deeply affect the PG in the strongly nonlinear regime. Hence, a correct description of $\mathrm{PG}$ in such a regime must keep such terms in the NLSE (1). Let us introduce a normalized noise field $u$ so that

$$
a_{r, i}=\sqrt{\frac{N N_{\mathrm{a}}}{2}} u_{r, i}
$$


and a parameter $\sigma \triangleq P \sqrt{\kappa /(4 \Delta \nu \mathrm{OSNR})}$ so that the NLSE, with the inclusion of the quadratic noise terms, can be written in the time domain as

$$
\left\{\begin{array}{l}
\frac{\partial u_{r}}{\partial z}=-\frac{\beta_{2}}{2} \frac{\partial^{2} u_{i}}{\partial t^{2}}+2 \gamma \sigma u_{r} u_{i} e^{g(z)}+\hat{S}_{r} \\
\frac{\partial u_{i}}{\partial z}=\frac{\beta_{2}}{2} \frac{\partial^{2} u_{r}}{\partial t^{2}}-\left(2 P u_{r}+3 \sigma u_{r}^{2}+\sigma u_{i}^{2}\right) \gamma e^{g(z)}+\hat{S}_{i}
\end{array}\right.
$$

where $\hat{S}$ is the normalized ASE forcing term. The term $\sigma$ shows that the effect of the quadratic noise terms scales as the ratio between the power and the square root of the OSNR so that they become relevant either at a large $P$ or at a very small OSNR.

Since the system performance, as noted from (4), mainly depends on the in-phase component, we are interested in the PSD of $u_{r}$, which can be calculated from the Wiener-Kinchine theorem [15] as

$$
G_{r r}(z, \omega)=\lim _{T \rightarrow \infty} \frac{1}{T} \mathrm{E}\left\{\left|u_{r T}(z, \omega)\right|^{2}\right\}
$$

where the index $T$ indicates a truncated field within a window of width $T$ centered at $t=0$. For simplicity, we will drop the $T$ subscript whenever it is clear that we are dealing with the truncated field.

Since an exact evaluation of $G_{r r}(z, \omega)$ for all $\omega$ is too involved, we will focus on the calculation of $G_{r r}(z, \omega=0)$ only, which is sufficient to get to the sought power threshold formula.

We evaluate the in-phase PSD noise at $\omega=0$ by applying the regular perturbation (RP) method [16], [17] in $\sigma$ to (7). From Appendix I, the first-order RP solution is

$$
G_{r r}(z, 0) \cong G_{r r 0}(z, 0)+\sigma^{2} G_{r r 1}(z, 0)
$$

where $G_{r r 0}(z, 0)=1$ is the (normalized) unperturbed PSD obtained from the small-signal model while a new term $G_{r r 1}(z, 0)$ appears whose closed-form expression for long systems $(N \gg 1)$ working at zero inline residual dispersion is derived in Appendix I as

$$
G_{r r}(0)=1+\sqrt{\frac{2 \alpha}{\left|\beta_{2}\right|}} \frac{\Phi_{\mathrm{NL}}^{4}}{24 \kappa^{3} \Delta \nu \mathrm{OSNR}} \Lambda(N)
$$

where $\Phi_{\mathrm{NL}}=\gamma N \kappa P / \alpha$ is the average received nonlinear phase for dispersion maps in which the transmission fiber is much longer than its attenuation length $1 / \alpha$, and $\Lambda(N)$ is a function of $N$, monotonically increasing to 1 for large $N$, as given in (28). A more accurate but more cumbersome expression, valid even in the case of nonzero residual dispersion, is also derived in Appendix I. Equation (10) reveals some interesting features that can be summarized as follows.

1) The PSD at $\omega=0$ of the normalized $u_{r}$ is inversely proportional to the OSNR, thus the effect of the noise-noise beating terms becomes large for systems operating at a small OSNR such as very long systems employing FEC codes. The small-signal model would predict instead an in-phase PSD value not affected by PG.

2) The dependence on a nonlinear phase is quartic. This implies an extremely fast degradation of performance with launched power, which leads to a power threshold effect.

3) The PSD inflation at $\omega=0$ does not depend on the sign of $\beta_{2}$ of the transmission fiber. For systems with low $\beta_{2}$, the inflation is larger because of the increased efficiency of FWM among noise frequencies.

Regarding 2), we can reasonably use as a threshold value the transmitted power at which the in-phase PSD doubles with respect to the linear case (no PG). From (10), we find such an average power threshold to be

$$
P_{\mathrm{th}} \triangleq\left(\frac{24 \sqrt{\left|\beta_{2}\right|} \alpha^{\frac{7}{2}} \kappa^{3} \Delta \nu \mathrm{OSNR}}{\sqrt{2} \gamma^{4} N^{4} \Lambda(N)}\right)^{\frac{1}{4}}
$$

We tested our RP solution (10) as well as the more complete formula (25) against the numerical simulation of propagation in a periodic transmission system composed of $N$ identical compensated spans of $100 \mathrm{~km}$ of Teralight each and a given inline residual dispersion as in Fig. $2{ }^{2}$

We estimated the normalized in-phase ASE PSD by the averaged periodogram method [13] applied to long sequences of $2^{16}$ noise samples added to a $\mathrm{CW}$ signal and propagated along the line by the split-step Fourier method [1].

Fig. 4 (top) shows the normalized in-phase ASE PSD gain at $\omega=0$ with full inline compensation evaluated with the complete formula (25) (solid line), its approximation (10) (dashed line), and the periodogram-based simulations (circles) for two values of the end-line OSNR $=10 \mathrm{~dB}$ (filled circles) and $16 \mathrm{~dB}$ (open circles) in a Teralight-based five-span system. A good match is observed between the analytical formulas and the simulations up to a gain inflation of about $5 \mathrm{~dB}$ for both values of OSNR.

The bottom plot of Fig. 4 shows the 3 -dB power threshold, derived from the complete formula (25), versus the end-line OSNR at an inline residual dispersion per span of $0 \mathrm{ps} / \mathrm{nm}$ [solid line, coinciding with (11)], $+50 \mathrm{ps} / \mathrm{nm}$ (dashed line), and $+100 \mathrm{ps} / \mathrm{nm}$ (dashed-dotted line), while the symbols refer to the periodogram-based simulation. Besides the good match between theory and simulation, we note that a nonzero positive inline dispersion lowers the PG power threshold, although by only $1 \mathrm{~dB}$ in a wide range of $100 \mathrm{ps} / \mathrm{nm}$ per span.

\section{System Implications OF PG}

Since the formulas well reproduce the PSD inflation, in this section we investigate the impact of such an inflation on the BER of a dispersion-managed system.

We performed numerical simulations of the $15 \times 100 \mathrm{~km}$ 10-Gb/s experimental system shown in Fig. 2, but now we allowed the transmission fiber to be either Teralight or $\mathrm{NZDSF}^{+}$

\footnotetext{
${ }^{2}$ Note that the presence of linear pre- and postcompensation fibers does not change the PSDs at $\omega=0$ since there is no GVD-induced phase rotation at $\omega=0$.
} 

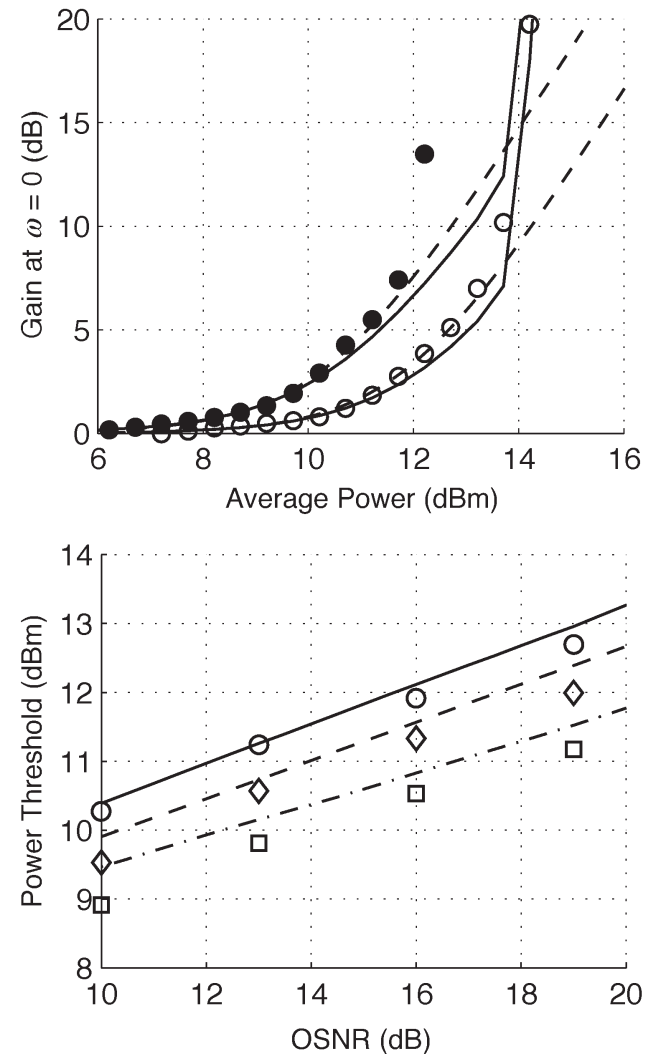

Fig. 4. Top: Normalized in-phase ASE PSD gain at $\omega=0$ versus power evaluated at an end-line OSNR $=10 \mathrm{~dB}$ (filled circles) and $16 \mathrm{~dB}$ (open circles) by the periodogram-based simulations and by the complete formula (25) (solid line) and its approximation (27) (dashed line) for a Teralight system. Bottom: 3-dB power threshold versus OSNR with inline dispersion per span of $0 \mathrm{ps} / \mathrm{nm}$ (solid line), $+50 \mathrm{ps} / \mathrm{nm}$ (dashed line), and $+100 \mathrm{ps} / \mathrm{nm}$ (dashed-dotted line). Symbols: Simulation.

(non-zero dispersion shifted fiber, $D=2.9 \mathrm{ps} / \mathrm{nm} / \mathrm{km}$, $\gamma=2.1 \mathrm{~W}^{-1} \mathrm{~km}^{-1}$ ) or SMF (single-mode fiber, $D=$ $17 \mathrm{ps} / \mathrm{nm} / \mathrm{km}, \gamma=1.4 \mathrm{~W}^{-1} \mathrm{~km}^{-1}$ ). For the $\mathrm{NZDSF}^{+}$and the SMF fibers, we set the inline residual dispersion to zero and optimized the pre- and postcompensation fibers for each transmitted power so as to obtain the lowest simulated BER in the presence of SPM but in the absence of PG. The receiver had a Gaussian optical filter of bandwidth $0.23 \mathrm{~nm}$ and an electrical second-order Butterworth filter of bandwidth 6.5 GHz. We transmitted a $2^{6}-1$ PRBS at different powers. As in the experiment, the OSNR was uniformly degraded at each amplifier along the line to get the desired end-line OSNR while the desired BER at the receiver was reached by changing the preamplifier noise figure through a variable attenuator. BER was estimated by the semianalytical method.

All results will be expressed in terms of the average nonlinear phase, which is used in the optimization of single-channel dispersion-mapped transmission systems impaired by SPM [18] since performance scales as the product of transmitted power and number of spans. The PG nonlinear phase threshold $\Phi_{\mathrm{th}}=$ $\gamma N P_{\text {th }} / \alpha \propto \Lambda(N)^{-1 / 4}$ obtained by using (11) converges to a fixed value with an increasing number of spans, as shown for instance in Fig. 5, which refers to an $\mathrm{NZDSF}^{+}$-based system with an end-line OSNR of $16 \mathrm{~dB}$. From the figure, we observe that $\Phi_{\text {th }}$ nearly stabilizes after ten spans since the system tends

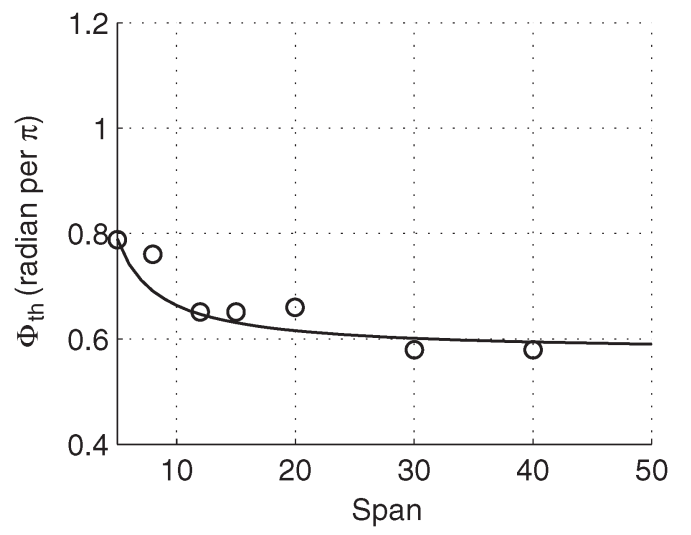

Fig. 5. Nonlinear phase threshold versus number of spans. $\mathrm{NZDSF}^{+}$-based system with end-line OSNR $=16 \mathrm{~dB}$ and zero inline residual dispersion. Solid line: analytical formula. Circles: simulation.

to look like a distributed system. Hence, when $N$ is large, even in the presence of $\mathrm{PG}$, the performance still scales with the nonlinear phase.

We measured the received OSNR penalty with respect to back-to-back BER $=10^{-5}$ for a signal distorted by SPM and group velocity dispersion (GVD) and with either white ASE or PG-colored ASE so as to measure the relative impact of PG. Fig. 6 shows such an OSNR penalty (top graphs) versus nonlinear phase for the three different transmission fiber types. With PG, the end-line OSNR was fixed at either 16 or $19 \mathrm{~dB}$. The bottom row of Fig. 6 shows the corresponding PG-induced in-phase ASE PSD inflation as obtained by (10), which clearly demonstrates the correlation between the in-phase ASE PSD inflation and the disruption of system performance with the increase of the nonlinear phase for all transmission fiber types.

We note the large difference of $1.5 \pi$ radians between the PG threshold and the SPM threshold (i.e., the $\Phi_{\mathrm{NL}}$ at which $\mathrm{SPM}+\mathrm{GVD}+$ white ASE cause an error floor) in the $\mathrm{NZDSF}^{+}$ case, while for Teralight and SMF such a difference is much reduced.

We also report the experimental points for the Teralight case already given in Fig. 3, whose reasonable match with the simulation is a good indicator of the validity of our semianalytical BER evaluation method.

We repeated the simulations for the Teralight-based system in the absence of the final preamplifier so that the end-line OSNR coincides with the received OSNR. Fig. 7 (top) shows the contour plot of the BER versus the OSNR and the average nonlinear phase both without (dashed lines) and with (solid lines) PG. The dashed line indicated by (a) shows the PG nonlinear phase threshold as obtained from (11), which well delimits the region of strongly nonlinear PG. We note that, at small nonlinear phases well below the PG threshold, the systems with PG have a slightly lower BER than those without PG because of the squeezing of the in-phase ASE in the optimized map.

Fig. 7 (bottom) shows on the same plane the contour plot of the OSNR penalty of the system with PG relative to the system without PG at equal BER. Clearly, to the right of curve (a), penalties due to PG become significant from a system perspective and lead to system disruption when the OSNR is small. 

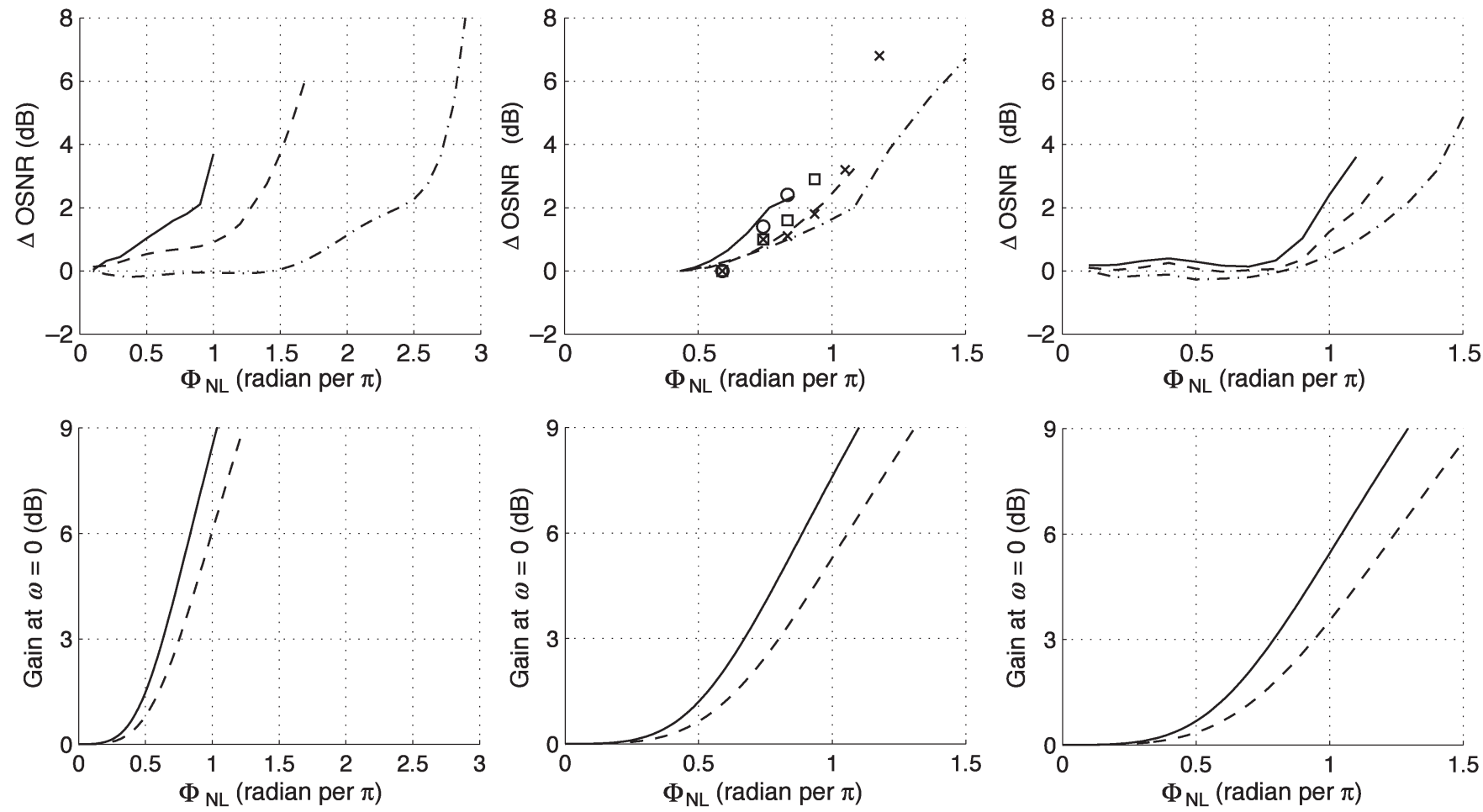

Fig. 6. Received OSNR penalty with respect to the back-to-back BER $=10^{-5}$ (top row) and in-phase ASE PSD gain at $\omega=0$ (bottom row) versus an average nonlinear phase for an NZDSF ${ }^{+}$(left), Teralight (center), and SMF-based (right) 15-span 10-Gb/s system including PG at end-line OSNR of 16 dB (solid) and 19 dB (dashed). Curves without PG: Dashed-dotted line. Top center: Symbols indicate the experimental results (same points as in Fig. 3).

The figure can also be used to prove that PG in the strongly nonlinear regime does have a significant impact in long-haul systems employing FECs, which are operated at a very small OSNR. For instance, in Fig. 7 (top), curve (b) in bold line represents the OSNR, derived from (2), for the same dispersionmapped system extended to $N=60$ spans and with a noise figure per span of $5.5 \mathrm{~dB}$. The points on the curve correspond to different launched powers. We note that, while by assuming the received noise as white one would conclude that the system can work at a BER $<10^{-5}$ for $\Phi_{\mathrm{NL}}>0.7 \pi$, with the realistic inclusion of PG such a conclusion is clearly false.

The additional penalty introduced by PG can be compensated for in two alternative ways, as it can be deduced from Fig. 8, which shows for instance the BER evaluated at $\Phi_{\mathrm{NL}}=0.75 \pi$ [drawn from the contour in Fig. 7 (top)] both without (circles) and with (diamonds) PG. The dashed lines show the corresponding coded BER for a standard (255 239) Reed-Solomon code [19]. Suppose that one wishes to operate the system at an uncoded BER of $10^{-5}$, corresponding to a coded BER of about $10^{-22}$. Then, when neglecting $\mathrm{PG}$, one finds from the open circle solid curve that an OSNR $=13.5 \mathrm{~dB}$ should be used. However, PG worsens the BER to about $5 \times 10^{-4}$ in the diamond curve case, leading to a coded BER of about $10^{-8}$. The first technique to restore the desired coded BER is by working at the same OSNR and using a more powerful code, whose additional coding gain at $\mathrm{BER}=10^{-8}$ should roughly be $\Delta \mathrm{G} \sim 2.2 \mathrm{~dB}$, as shown in the figure. The second way is by keeping the same FEC but increasing the OSNR by $\triangle$ OSNR $\sim 1.5 \mathrm{~dB}$ [a value that can also be read off Fig. 7 (bottom)] so as to leave the uncoded BER at $10^{-5}$.
Note that OSNR/coding gain penalties are expected to be much larger for transmission fibers with a lower dispersion than Teralight, as inferred from the OSNR curves in Fig. 6.

As for the limits of our semianalytical BER evaluation method, a critical assumption is that the received ASE noise statistics remain Gaussian even after nonlinear propagation. However, in the same simulations used to estimate the ASE PSD, we also verified that the histograms estimating the true joint pdf (jpdf) of the in-phase and quadrature noise components, for nonlinear phase values up to the power threshold, reasonably resemble a Gaussian jpdf, characterized by elliptical contours. As the nonlinear phase is increased further, the true jpdf contours tend to bend, as shown in the left column of Fig. 9 (top), which also shows (bottom) the Gaussian jpdf having the true covariance matrix estimated by the simulation, i.e., the one used by our semianalytical method. From the jpdf's, we evaluated the cumulative distribution function (CDF) of the sampled current $Y$ on marks, i.e., the probability that $Y$ is lower than the decision threshold $\theta$, which we plot in the right graph of Fig. 9. Although the Gaussian jpdf fails to capture the bending of the exact jpdf, even at a nonlinear phase value of $0.75 \pi$, slightly exceeding the PG threshold, there is still a reasonable match between the exact (a) and the Gaussian-approximated (b) CDF, with a slight underestimation by the Gaussian approximation. Finally, curve (c) reports the Gaussian-approximated CDF that uses the covariance matrix obtained from the small-signal PG model, which largely underestimates the exact CDF. The underestimation of the BER by our semianalytical method beyond the PG threshold can also be observed in Fig. 6, in which a good match between experiment and analysis is found for nonlinear phases up to PG 

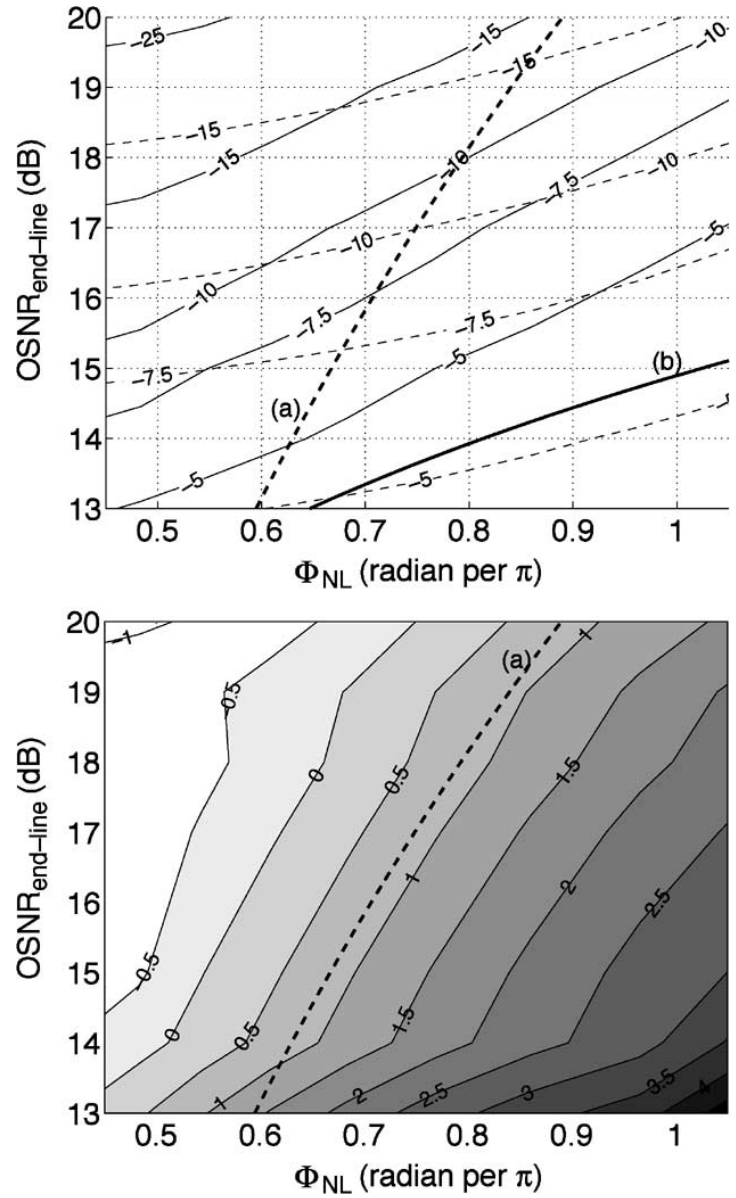

Fig. 7. Top: contour plot of $\log _{10}$ (BER) versus an end-line OSNR and an average nonlinear phase for the Teralight-based 15-span system in the absence of PG (dashed lines) and with PG (solid lines). (a) PG nonlinear phase threshold $\Phi_{\text {th }}$. (b) OSNR from (2) for the same map with $N=60$ spans and a noise figure per span of $5.5 \mathrm{~dB}$. Bottom: OSNR penalty (decibel) versus OSNR and the average nonlinear phase.

threshold. Beyond threshold, experimental penalties are seen to grow faster with nonlinear phase than those predicted by our method.

\section{CONCLUSION}

In this paper, we have proved for the first time, both experimentally and numerically, that parametric gain $(\mathrm{PG})$ is a nonnegligible factor in the design and analysis of long-haul dispersion-managed optical 10-Gb/s ON-OFF keying (OOK) nonreturn-to-zero (NRZ) transmission systems operated at "small" optical signal-to-noise ratio (OSNR) values, a regime in which the amplified spontaneous emission (ASE)-ASE beating along the fiber cannot be neglected.

Although the complete stochastic analysis of the propagation equations is prohibitively complex, we managed to derive meaningful analytical results by using perturbative techniques to solve the nonlinear Schrödinger equation (NLSE) and by concentrating on the power spectral density (PSD) of the inphase ASE noise at the carrier frequency. We proved that the PG-induced inflation of such a PSD for increasing values of the average nonlinear phase is strongly correlated with the

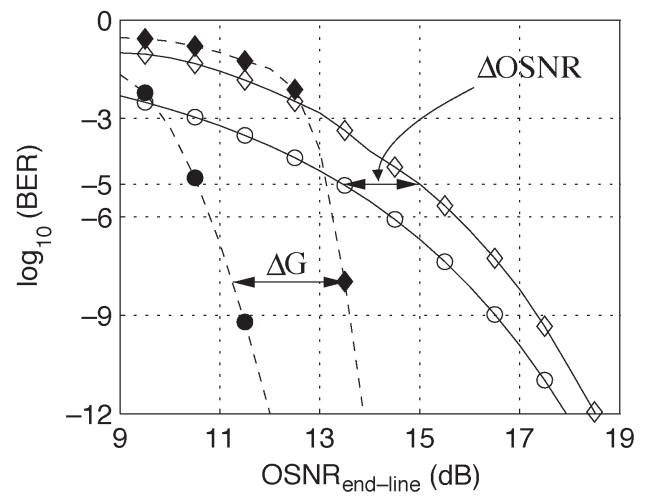

Fig. 8. Uncoded BER (solid lines) and coded BER (dashed lines) versus an end-line OSNR for a Teralight map. Diamonds: SPM + PG; Circles: SPM + white noise. $\Phi_{\mathrm{NL}}=0.75 \pi$. $\mathrm{N}=15$ spans.
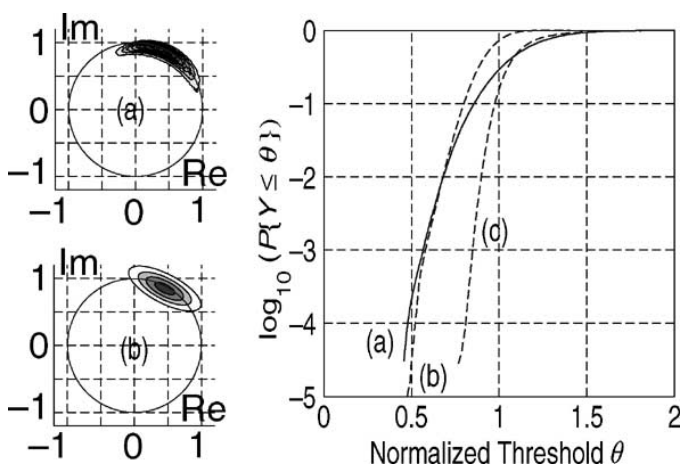

Fig. 9. (Left) jpdf of the received optical field (after optical filter) obtained by (a) Monte Carlo simulation and (b) its Gaussian fit with exact covariance matrix. (Right) $\mathrm{CDF}$ of the sampled current $Y$ in cases (a) and (b). Case (c) is the same as (b), but the covariance is obtained from a small-signal model. $15 \times 100 \mathrm{~km}$ Teralight system, $30 \mathrm{ps} / \mathrm{nm} / \mathrm{span}$ inline dispersion, optimized pre- and postcompensation, $\Phi_{\mathrm{NL}}=0.75 \pi$, end-line OSNR $=16 \mathrm{~dB}$.

corresponding inflation of the required OSNR to achieve a given bit error rate (BER) level. We derived a very simple formula for the nonlinear phase threshold beyond which the system designer should not neglect the ASE-ASE beating along the transmission line when deriving the system BER. Such a formula can also be used to judge when the OSNR is "small" enough that PG becomes significant.

We have shown that a BER evaluation method that includes the ASE-ASE beating during propagation is mandatory to assess the effective gain of forward-error corrections (FECs) in long-haul $10-\mathrm{Gb} / \mathrm{s}$ systems operated at small OSNR. We verified that more than $1 \mathrm{~dB}$ of extra coding gain may be required when operating the system beyond the PG threshold with respect to what one can plan when evaluating system performance by ignoring the ASE-ASE beating along the line.

Note from Fig. 6 (top) that when increasing the fiber dispersion, the self-phase modulation (SPM) threshold tends to get closer to the PG power threshold and eventually becomes smaller so that performance is eventually limited by SPM instead of PG. Since performance scales as the product of bit rate squared times fiber dispersion, then increasing the bit rate by a factor of 4 has the same effect as increasing the dispersion 
by a factor of 16 . Hence, moving from 10 to $40 \mathrm{~Gb} / \mathrm{s}$, we expect the SPM to become the dominant impairment, and one needs not to worry about PG anymore.

\section{APPENDIX I}

\section{A. Perturbative Approach}

In this Appendix, we evaluate the PSD of $u_{r}(z, \omega=0)$ through a perturbative approach. Consider the $N$-span system described in Fig. 2. We note that the pre- and postcompensating fibers can be neglected in the analysis since they usually operate in the low power regime; therefore, that they cannot alter the received ASE PSD at our target frequency $\omega=0$.

The terms proportional to $\sigma$ in (7) are a small perturbation to the equation, so we search a perturbed solution in the form

$$
\left\{\begin{array}{l}
u_{r}(z, \omega)=u_{r 0}(z, \omega)+\sigma u_{r 1}(z, \omega)+\mathrm{O}\left(\sigma^{2}\right) \\
u_{i}(z, \omega)=u_{i 0}(z, \omega)+\sigma u_{i 1}(z, \omega)+\mathrm{O}\left(\sigma^{2}\right)
\end{array}\right.
$$

where $\mathrm{O}\left(\sigma^{2}\right)$ represents higher order terms in $\sigma$, while the unperturbed field $u_{r, i 0}$ and the perturbed one $\sigma u_{r, i 1}$ can be found by substituting (12) in (7) and equating matching powers in $\sigma$ [16]. Note that $u_{r, i 0}$ is the solution of the small-signal model in (5). Even if the expression of $u_{r 1}$ is quite involved, at $\omega=0$ it takes the simple form

$$
u_{r 1}(z, 0)=2 \gamma \int_{0}^{z} \mathrm{e}^{g(\zeta)} F(\zeta) \mathrm{d} \zeta
$$

where

$$
\left.F(z) \triangleq \frac{1}{2 \pi} u_{r 0}(z, \omega) \otimes u_{i 0}(z, \omega)\right|_{\omega=0}
$$

and $\otimes$ denotes convolution in $\omega$. We can now approximate the PSD of $u_{r}$ as

$$
G_{r r}(z, 0) \simeq \lim _{T \rightarrow \infty} \frac{1}{T} \mathrm{E}\left\{\left|u_{r 0}(z, 0)+\sigma u_{r 1}(z, 0)\right|^{2}\right\} .
$$

From (13) and (14), we infer that

$$
\mathrm{E}\left\{u_{r 0}(z, 0) u_{r 1}^{*}(z, 0)\right\}=\mathrm{E}\left\{u_{r 0}^{*}(z, 0) u_{r 1}(z, 0)\right\}=0
$$

since they involve third moments of jointly Gaussian random variables of zero mean [20]. The PSD of $u_{r}$ is therefore the sum of the unperturbed PSD of $u_{r 0}$, which we indicate as $G_{r r 0}$, and a perturbative term $\sigma^{2} G_{r r 1}{ }^{3}$

$$
G_{r r}(z, 0)=G_{r r 0}(z, 0)+\sigma^{2} G_{r r 1}(z, 0) .
$$

\footnotetext{
${ }^{3}$ Note that the term $G_{r r 0}(z, 0)$ comes from $u_{r 0}(z, \omega)$ while the term $\sigma^{2} G_{r r 1}(z, 0)$ comes from $\sigma u_{r 1}(z, \omega)$. However, if we also include $\sigma^{2} u_{r 2}(z, \omega)$ in the expansion of $u_{r}(z, \omega)$, then the term $u_{r 0}(z, \omega) \sigma^{2} u_{r 2}(z$ $\omega)$ contributes an extra term of order $\sigma^{2}$ to $G_{r r}(z, 0)$. In the following, we will ignore such a contribution, which is as hard to compute as the overall PSD. In the results section, we check against simulation that (15) well matches the "exact" PSD at $\omega=0$, and therefore we conclude a posteriori that the contribution of the terms that we overlooked, at the tested values of OSNR, is negligible.
}

Let us consider the unperturbed PSD first. The solution of (5) can be expressed in terms of a transfer matrix $\mathbf{H}(z, s, \omega)$ [5] that accounts for propagation from $s$ to $z$ at frequency $\omega$, so that within the $m$ th span, i.e., for $z_{m-1}<z<z_{m}$, we can write

$$
\left[\begin{array}{l}
a_{r}(z, \omega) \\
a_{i}(z, \omega)
\end{array}\right]=\sum_{k=1}^{m-1} \mathbf{H}\left(z, z_{k}, \omega\right)\left[\begin{array}{l}
n_{r}\left(z_{k}, \omega\right) \\
n_{i}\left(z_{k}, \omega\right)
\end{array}\right]
$$

with $n\left(z_{k}, \omega\right)$ being the noise field generated by the $k$ th lumped amplifier, and we assume a noiseless source. The unperturbed PSD can be evaluated from the theory of linear systems [20] applied to (16). However, from (5), we immediately observe that at the reference frequency $\omega=0$ the in-phase noise component is invariant along $z$, and so is its unperturbed PSD, i.e., $G_{r r 0}(z, 0)=1$ for all $z$.

The perturbed PSD is proportional to

$$
\begin{aligned}
& G_{r r 1}(z, 0) \triangleq \lim _{T \rightarrow \infty} \frac{1}{T} \mathrm{E}\left\{\left|u_{r 1}(z, 0)\right|^{2}\right\} \\
&=\int_{0}^{z} \int_{0}^{z} \mathrm{e}^{g(\zeta)+g(\mu)} \lim _{T \rightarrow \infty} \frac{4 \gamma^{2}}{T} \\
& \quad \times \mathrm{E}\left\{F(\zeta) F^{*}(\mu)\right\} \mathrm{d} \zeta \mathrm{d} \mu
\end{aligned}
$$

where

$$
\begin{aligned}
\mathrm{E} & \left\{F(\zeta) F^{*}(\mu)\right\} \\
& =\iint \mathrm{E}\left\{u_{r 0}(\zeta, \lambda) u_{i 0}(\zeta,-\lambda) u_{r 0}^{*}(\mu, \eta) u_{i 0}^{*}(\mu,-\eta)\right\} \frac{\mathrm{d} \lambda}{2 \pi} \frac{\mathrm{d} \eta}{2 \pi} .
\end{aligned}
$$

In order to evaluate (18), we define the PSD matrix

$$
\mathbf{G}(\zeta, \mu, \omega) \triangleq\left[\begin{array}{ll}
G_{r r 0}(\zeta, \mu, \omega) & G_{r i 0}(\zeta, \mu, \omega) \\
G_{i r 0}(\zeta, \mu, \omega) & G_{i i 0}(\zeta, \mu, \omega)
\end{array}\right]
$$

whose elements are the unperturbed PSDs of the kind $G_{x y 0}(\zeta, \mu, \omega) \triangleq \lim _{T \rightarrow \infty}(1 / T) \mathrm{E}\left\{u_{x 0}(\zeta, \omega) u_{y 0}^{*}(\mu, \omega)\right\},(x, y)$ $\in(r, i) .{ }^{4}$ In Appendix II, we show that

$$
\begin{aligned}
& \lim _{T \rightarrow \infty} \frac{\mathrm{E}\left\{F(\zeta) F^{*}(\mu)\right\}}{T} \\
&=\int\left[G_{r r 0}(\zeta, \mu, \lambda) G_{i i 0}(\zeta, \mu, \lambda)\right. \\
&\left.\quad \quad+G_{r i 0}(\zeta, \mu, \lambda) G_{i r 0}(\zeta, \mu,-\lambda)\right] \frac{\mathrm{d} \lambda}{2 \pi} \\
& \triangleq \int w(\zeta, \mu, \lambda) \mathrm{d} \lambda .
\end{aligned}
$$

The elements of $\mathbf{G}(\zeta, \mu, \omega)$, which are building blocks of the integrand $w(\zeta, \mu, \lambda)$, can be calculated as follows.

\footnotetext{
${ }^{4}$ When $\zeta \equiv \mu$, we prefer to write such coordinate only once, as for instance in $G_{r r 0}(z, 0)$.
} 
If $\zeta \in n$th span and $\mu \in m$ th span, since all the $n\left(z_{k}, t\right)$ noise terms are i.i.d. with zero mean, from (16) and the definition of $\mathbf{G}(\zeta, \mu, \omega)$ we get

$$
\mathbf{G}^{n m}(\zeta, \mu, \omega)=\frac{1}{N} \sum_{k=1}^{\min (n, m)-1} \mathbf{H}\left(\zeta, z_{k}, \omega\right) \mathbf{H}^{\mathrm{T}}\left(\mu, z_{k}, \omega\right)
$$

where $1 / N$ is the (normalized) PSD of the ASE noise of one amplifier, and we emphasized the dependence on $n$ and $m$ by adding the index $n m$. For an optical system composed of identical dispersion managed spans of length $L$, we have $\mathbf{H}\left(z_{k+1}, z_{k}, \omega\right)=\mathbf{H}(L, 0, \omega) \triangleq \mathbf{H}_{L}(\omega)$, so that (21) becomes

$$
\begin{aligned}
& \mathbf{G}^{n m}(\zeta, \mu, \omega)=\mathbf{H}(\zeta,\left.z_{n-1}, \omega\right) \frac{1}{N} \sum_{k=1}^{\min (n, m)-1} \mathbf{H}_{L}^{n-k-1} \\
& \times\left(\mathbf{H}_{L}^{T}\right)^{m-k-1} \mathbf{H}^{T}\left(\mu, z_{m-1}, \omega\right) .
\end{aligned}
$$

For a given total nonlinear phase rotation, when the number of spans is sufficiently large the nonlinear phase rotation per span is small, so that we can approximate the local propagation matrix $\mathbf{H}\left(z, z_{k-1}, \omega\right), z_{k-1}<z<z_{k}$ in (22) with its expression in the presence of GVD only

$$
\begin{aligned}
& \mathbf{H}\left(z, z_{k-1}, \omega\right) \\
& \simeq\left[\begin{array}{cc}
\cos \left(\omega^{2} \int_{z_{k-1}}^{z} \frac{\beta_{2}(\eta)}{2} \mathrm{~d} \eta\right) & \sin \left(\omega^{2} \int_{z_{k-1}}^{z} \frac{\beta_{2}(\eta)}{2} \mathrm{~d} \eta\right) \\
-\sin \left(\omega^{2} \int_{z_{k-1}}^{z} \frac{\beta_{2}(\eta)}{2} \mathrm{~d} \eta\right) & \cos \left(\omega^{2} \int_{z_{k-1}}^{z} \frac{\beta_{2}(\eta)}{2} \mathrm{~d} \eta\right)
\end{array}\right]
\end{aligned}
$$

which becomes exact in the limit $N \rightarrow \infty$. We now define the matrix $\mathbf{T}^{n m}(\omega)$, whose elements we denote as $t_{i j}^{n m}$, as

$$
\mathbf{T}^{n m}(\omega) \triangleq \frac{1}{N} \sum_{k=1}^{\min (n, m)-1} \mathbf{H}_{L}^{n-k-1}\left(\mathbf{H}_{L}^{T}\right)^{m-k-1}
$$

so that the perturbed PSD $G_{r r 1}(z, 0)$, by using (22) in (20), yields (25), where the integrals in $\zeta$ and $\mu$ were solved by assuming that the transmission fiber length is much longer than its attenuation length $1 / \alpha$ and that the amplifiers exactly recover the span losses. Equation (25), which is displayed at the bottom of the page, can be rapidly evaluated by numerical integration.

\section{B. Approximate Formula}

We observe from (25) that the principal contribution to $G_{r r 1}(z, 0)$ comes from the first integral in the square brackets whose integrand is a very sharp low-pass filtering [of corner frequency $\left.\lambda_{0}=(1 / 2 \pi) \sqrt{\left(\alpha /\left|\beta_{2}\right|\right)}\right]$ of the entries of matrix $\mathbf{T}^{n m}$. If $\mathbf{H}_{L}(\omega)$ is a slowly varying function of $\omega$ around $\omega=0$ up to such a corner frequency, we can approximate $G_{r r 1}(z, 0)$ as

$$
\begin{aligned}
G_{r r 1}(z, 0) & \simeq \gamma^{2} \frac{3 \sqrt{2}}{4 \sqrt{\left|\beta_{2}\right|} \alpha^{\frac{3}{2}}} \\
& \times \sum_{n=1}^{N} \sum_{m=1}^{N}\left(t_{11}^{n m}(0) t_{22}^{n m}(0)+t_{12}^{n m}(0) t_{21}^{n m}(0)\right) .
\end{aligned}
$$

The case that best meets the above requirement on $\mathbf{H}_{L}(\omega)$ occurs when the inline dispersion is zero, since it can be proven that in such a case the Taylor expansion of $\mathbf{H}_{L}(\omega)=\mathbf{H}_{L}(0)+$ $\mathbf{H}_{L}^{(2)}(0) \omega^{2} / 2+\mathbf{H}_{L}^{(4)}(0) \omega^{4} / 4 !+\ldots$ has a null matrix coefficient of the term in $\omega^{2}$.

Now, since $\mathbf{H}_{\mathbf{L}}(0)=\left[\begin{array}{cc}1 & 0 \\ -2 \gamma P / \alpha & 1\end{array}\right]$, we can evaluate $\mathbf{T}^{n m}(0)$ in (24) and close the double sum in (26). For $N \gg 1$, as required for approximation (23) to be valid, we obtain

$$
G_{r r}(0) \simeq 1+\frac{\sqrt{2} \gamma^{4} P^{4} N^{4} \kappa}{24 \Delta \nu \operatorname{OSNR} \sqrt{\left|\beta_{2}\right|} \alpha^{\frac{7}{2}}} \Lambda(N)
$$

where

$$
\Lambda(N) \triangleq \frac{(N-1)(N-2)\left(20 N^{3}-48 N^{2}+31 N-21\right)}{20 N^{5}}
$$

$$
\begin{aligned}
G_{r r 1}(z, 0)=4 \gamma^{2} \int_{-\infty}^{+\infty} \int_{0}^{z} \int_{0}^{z} e^{g(\zeta)+g(\mu)} w(\zeta, \mu, \lambda) \mathrm{d} \zeta \mathrm{d} \mu \mathrm{d} \lambda \\
=4 \gamma^{2} \sum_{n=1}^{N} \sum_{m=1}^{N}\left[\int_{-\infty}^{+\infty} \frac{\alpha^{2}}{\left(\alpha^{2}+16 \pi^{4} \beta_{2}^{2} \lambda^{4}\right)^{2}}\left(t_{11}^{n m}(\lambda) t_{22}^{n m}(\lambda)+t_{12}^{n m}(\lambda) t_{21}^{n m}(\lambda)\right) \mathrm{d} \lambda\right. \\
+\int_{-\infty}^{+\infty} \frac{4 \pi^{2} \alpha \beta_{2} \lambda^{2}}{\left(\alpha^{2}+16 \pi^{4} \beta_{2}^{2} \lambda^{4}\right)^{2}}\left[\left(t_{21}^{n m}(\lambda)+t_{12}^{n m}(\lambda)\right)\left(t_{22}^{n m}(\lambda)-t_{11}^{n m}(\lambda)\right)\right] \mathrm{d} \lambda \\
\left.\quad+\int_{-\infty}^{+\infty} \frac{8 \pi^{4} \beta_{2}^{2} \lambda^{4}}{\left(\alpha^{2}+16 \pi^{4} \beta_{2}^{2} \lambda^{4}\right)^{2}}\left(\left(t_{11}^{n m}(\lambda)\right)^{2}+\left(t_{22}^{n m}(\lambda)\right)^{2}-\left(t_{12}^{n m}(\lambda)\right)^{2}-\left(t_{21}^{n m}(\lambda)\right)^{2}\right) \mathrm{d} \lambda\right]
\end{aligned}
$$


so that, by introducing the average nonlinear phase, (27) is written as in (10).

\section{APPENDIX II}

Consider the Gaussian real processes $x_{n}(t), n=1,2$, with zero mean and correlation functions $R_{i k}(\tau) \triangleq \mathrm{E}\left\{x_{i}(t) x_{k}(t+\right.$ $\tau)\}$, whose corresponding Fourier transforms are the PSD $G_{i k}(\omega)$. Let the gated Fourier transform of $x_{n}$ be $X_{n}(\omega)=$ $\int_{-T / 2}^{T / 2} x_{n}(t) e^{-j \omega t} \mathrm{~d} t$. In (18), we face the problem of calculating quantities of the kind

$$
\lim _{T \rightarrow+\infty} \frac{1}{T} \iint \mathrm{E}\left\{X_{1}(\lambda) X_{2}(-\lambda) X_{1}^{*}(\eta) X_{2}^{*}(-\eta)\right\} \mathrm{d} \lambda \mathrm{d} \eta .
$$

Equation (29) is thus a joint moment of order 4 of Gaussian processes. The calculation of the average value can be decomposed as the sum of products of second-order moments according to the Isserlis formula [21]

$$
\begin{aligned}
& \mathrm{E}\left\{X_{1}(\lambda) X_{2}(-\lambda) X_{1}^{*}(\eta) X_{2}^{*}(-\eta)\right\} \\
&= \mathrm{E}\left\{X_{1}(\lambda) X_{2}(-\lambda)\right\} \mathrm{E}\left\{X_{1}^{*}(\eta) X_{2}^{*}(-\eta)\right\} \\
&+\mathrm{E}\left\{X_{1}(\lambda) X_{1}^{*}(\eta)\right\} \mathrm{E}\left\{X_{2}(-\lambda) X_{2}^{*}(-\eta)\right\} \\
&+\mathrm{E}\left\{X_{1}(\lambda) X_{2}^{*}(-\eta)\right\} \mathrm{E}\left\{X_{2}(-\lambda) X_{1}^{*}(\eta)\right\} .
\end{aligned}
$$

The terms in the above equation are of the kind $\mathrm{E}\left\{X_{i}(\lambda) X_{k}^{*}(\eta)\right\}=\int_{T} \int_{T} R_{i k}\left(t_{1}-t_{2}\right) e^{-j\left(\lambda t_{1}-\eta t_{2}\right)} \mathrm{d} t_{1} \mathrm{~d} t_{2}$. After the change of variables $\tau \triangleq t_{1}-t_{2}, \theta \triangleq t_{1}$, we have

$$
\begin{gathered}
\mathrm{E}\left\{X_{i}(\lambda) X_{k}^{*}(\eta)\right\}=\int_{-T}^{0} R_{i k}(\tau) e^{-j \eta \tau}\left[\int_{-\frac{T}{2}}^{\tau+\frac{T}{2}} e^{-j(\lambda-\eta) \theta} \mathrm{d} \theta \mathrm{d} \tau\right] \\
+\int_{0}^{T} R_{i k}(\tau) e^{-j \eta \tau}\left[\int_{\tau-\frac{T}{2}}^{\frac{T}{2}} e^{-j(\lambda-\eta) \theta}\right] \mathrm{d} \theta \mathrm{d} \tau .
\end{gathered}
$$

Note that in the limit $T \rightarrow+\infty$, the terms in square brackets become delta functions, so that $\lim _{T \rightarrow+\infty} \mathrm{E}\left\{X_{i}(\lambda) X_{k}^{*}(\eta)\right\}=$ $2 \pi G_{i k}(\lambda) \delta(\lambda-\eta)$. Dividing (31) by $T$ and taking the limit, we finally obtain the PSD as given by the Wiener-Kinchine theorem

$$
\lim _{T \rightarrow+\infty} \frac{1}{T} \mathrm{E}\left\{X_{i}(\lambda) X_{k}^{*}(\eta)\right\}= \begin{cases}G_{i k}(\lambda) & \lambda=\eta \\ 0 & \lambda \neq \eta\end{cases}
$$

Since the limit (32) is finite, using (30) we finally get

$$
\begin{aligned}
\lim _{T \rightarrow+\infty} \frac{1}{T} \iint & \mathrm{E}\left\{X_{1}(\lambda) X_{2}(-\lambda) X_{1}^{*}(\eta) X_{2}^{*}(-\eta)\right\} \mathrm{d} \lambda \mathrm{d} \eta \\
& =2 \pi \int\left[G_{11}(\lambda) G_{22}(\lambda)+G_{12}(\lambda) G_{21}^{*}(-\lambda)\right] \mathrm{d} \lambda
\end{aligned}
$$

\section{REFERENCES}

[1] G. P. Agrawal, Nonlinear Fiber Optics, 2nd ed. San Diego, CA: Academic, 1995.

[2] J. Hansyrd, P. A. Andrekson, M. Westlund, J. Li, and P. Hedekvist, "Fiberbased optical parametric amplifiers and their applications," IEEE J. Quantum Electron., vol. 8, no. 3, pp. 506-520, May/Jun. 2002.

[3] C. J. McKinstrie, S. Radic, and A. R. Chraplyvy, "Parametric amplifiers driven by two pump waves," IEEE J. Quantum Electron., vol. 8, no. 3, pp. 538-547, May/Jun. 2002.

[4] M. Karlsson, "Modulation instability in lossy optical fibers," J. Opt. Soc. Amer. B, vol. 12, no. 11, pp. 2071-2078, Nov. 1995.

[5] A. Carena, V. Curri, R. Gaudino, P. Poggiolini, and S. Benedetto, "New analytical results on fiber parametric gain and its effects on ASE noise," IEEE Photon. Technol. Lett., vol. 9, no. 4, pp. 535-537, Apr. 1997.

[6] G. Bosco, A. Carena, V. Curri, R. Gaudino, P. Poggiolini, and S. Benedetto, "A novel approach to the evaluation of the impact of fiber parametric gain on the bit error rate," IEEE Trans. Commun., vol. 49, no. 12, pp. 2154-2163, Dec. 2001.

[7] C. W. Helstrom, "Distribution of the filtered output of a quadratic rectifier computed by numerical contour integration," IEEE Trans. Inf. Theory, vol. IT-32, no. 7, pp. 450-463, Jul. 1986.

[8] R. Holzlöhner, V. S. Grigoryan, C. R. Menyuk, and W. L. Kath, "Accurate calculation of eye diagrams and bit error rates in optical transmission systems using linearization," J. Lightw. Technol., vol. 20, no. 3, pp. 389400, Mar. 2002.

[9] D. Marcuse, "Single-channel operation in very long nonlinear fibers with optical amplifiers at zero dispersion," J. Lightw. Technol., vol. 9, no. 3, pp. 356-361, Mar. 1991.

[10] P. Serena and A. Bononi, "Power threshold due to parametric gain in dispersion-mapped communication systems," IEEE Photon. Technol. Lett., vol. 14, no. 11, pp. 1521-1523, Nov. 2002.

[11] E. Forestieri, "Evaluating the error probability in lightwave systems with chromatic dispersion, arbitrary pulse shape and pre- and postdetection filtering," J. Lightw. Technol., vol. 18, no. 11, pp. 1493-1503, Nov. 2000.

[12] P. Serena, "Studio dell'interazione tra segnale e rumore nella progettazione di sistemi ottici WDM in regime non lineare," Ph.D. thesis, Università degli Studi di Parma, Parma, Italy, Jan. 2003.

[13] A. V. Oppenheim and R. W. Schafer, Discrete-Time Signal Processing, 2nd ed. Upper Saddle River, NJ: Prentice-Hall, 1999.

[14] D. Pennickx, G. Charlet, J.-C. Antona, and L. Noirie, "Experimental validation of a transparent waveband-based optical backbone network," presented at the Eur. Conf. Optical Communication (ECOC), Copenhagen, Denmark, Sep. 2002, Paper 6.4.4.

[15] C. W. Helstroem, Elements of Signal Detection and Estimation. Englewood Cliffs, NJ: Prentice-Hall, 1995.

[16] D. Zwillinger, Handbook of Differential Equations. Boston, MA: Academic, 1989.

[17] A. Vannucci, P. Serena, and A. Bononi, "The RP method: A new tool for the iterative solution of the nonlinear Schrödinger equation," J. Lightw. Technol., vol. 20, no. 7, pp. 1102-1112, Jul. 2002.

[18] J.-C. Antona, S. Bigo, and J.-P. Faure, "Nonlinear cumulated phase as a criteria to assess performance of terrestrial WDM systems," in Proc. Optical Fiber Communication (OFC), Anaheim, CA, Mar. 2002, pp. 365-367.

[19] D. J. Torrieri, "The information-bit error rate for block codes," IEEE Trans. Commun., vol. COM-32, no. 4, pp. 474-476, Apr. 1984.

[20] A. Papoulis, Probability, Random Variables and Stochastic Processes, 3rd ed. New York: McGraw-Hill, 1991.

[21] W. A. Gardner, Introduction to Random Processes With Applications to Signals and Systems, 2nd ed. New York: McGraw-Hill, 1990, pp. 114-115.

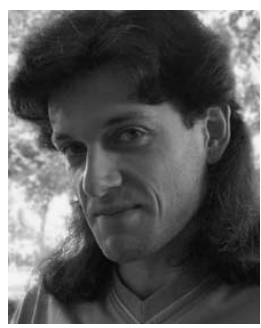

Paolo Serena was born in Piacenza, Italy, on 1973 $\mathrm{He}$ received the degree in electronic engineering and the $\mathrm{Ph} . \mathrm{D}$. degree in information technology for work on the nonlinear interaction between noise and signal in optical systems from the Università degli Studi di Parma, Parma, Italy, in 1999 and 2003, respectively.

His main research interests include the parametric gain of the amplified spontaneous emission (ASE) noise by nonlinear effects, phase-shifted modulation formats, and performance optimization in the nonlinear regime. 
Alberto Bononi, photograph and biography not available at the time of publication.

Jean-Christophe Antona, photograph and biography not available at the time of publication.

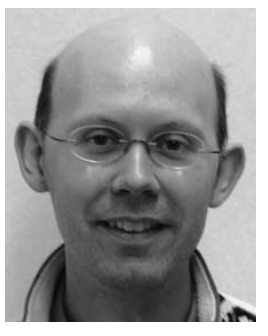

Sébastien Bigo (A'99-M'99) received the degree from the Ecole Supérieure d'Optique of Orsay, Orsay, France, in 1992 and the Ph.D. degree from the University of Besan, Besan, France, for work on all-optical signal processing for soliton undersea systems.

He joined Alcatel Research and Innovation, Marcoussis, France, where he is now head of the WDM Transmission Group, focusing on point-to-point terrestrial and submarine systems, as well as on transparent optical networks. His team has reported 15 transmission records out of large-scale WDM system demonstrators from 320 $\mathrm{Gb} / \mathrm{s}$ to $10 \mathrm{~Tb} / \mathrm{s}$. He holds 27 patents and has authored and coauthored 120 conference and journal papers, including 14 papers at the postdeadline sessions of OFC, ECOC, and OAA conferences. With three colleagues, he authored the reference book Erbium-Doped Fiber Amplifiers: Device and System Developments.

Dr. Bigo is a member of the Technical Committee of the IEEE Lasers \& Electro- Optics Society (LEOS) Annual Meeting. 